\title{
Dermal Organization in Scleroderma: The Fast Fourier Transform and the Laser Scatter Method Objectify Fibrosis in Nonlesional as well as Lesional Skin
}

\author{
Henry J.C. de Vries, Dory N.H. Enomoto, Jan van Marle, Paul P.M. van Zuijlen, \\ Jan R. Mekkes, and Jan D. Bos \\ Department of Dermatology (HJCdV, DNHE, JRM, JDB), and Department of Electron Microscopy (JVM), Academic \\ Medical Center, University of Amsterdam, and Department of Plastic, Reconstructive and Hand Surgery (PPMvZ), \\ Academic Medical Center, University of Amsterdam, Amsterdam, and Burns Center, Red Cross Hospital, Beverwijk, \\ The Netherlands
}

\begin{abstract}
SUMMARY: Scleroderma, a chronic, progressive disorder, is characterized by dermal fibrosis with collagen bundles orientated parallel to the epidermis. Simple objective parameters to evaluate disease progression and therapies are needed. We describe two methods, the laser scatter method and the fast Fourier transform (FFT), to measure collagen bundle orientation and spacing. Lesional sclerodermic skin (LS), nonlesional sclerodermic skin (nonLS), and control skin (CS) sections were evaluated for orientation ratio using the laser scatter method. The FFT was used to calculate orientation ratio, variation, and spacing of collagen bundles. Parameters were correlated with local and mean skin score measurements, on a scale of 0 (normal) to 3 (severely sclerotic). With both the laser scatter method and the FFT, orientation ratios of LS (respectively, $2.16 \pm 0.33$ and $1.83 \pm 0.62$ ) were significantly higher than CS (respectively, $1.70 \pm 0.35$ and $1.38 \pm 0.15$ ). NonLS orientation ratios (respectively, $1.92 \pm 0.15$ and $1.48 \pm 0.44$ ) were between LS and CS ratios. Orientation variation and bundle spacing of LS (respectively, $57.3 \pm 19.4$ and $15.7 \pm 5.6 \mu \mathrm{m}$ ) were significantly reduced compared to CS (respectively, $73.8 \pm 15.0$ and $18.9 \pm 1.9 \mu \mathrm{m}$ ). NonLS orientation ratios (respectively, $57.2 \pm 29.0$ and $15.6 \pm 6.1 \mu \mathrm{m}$ ) were similar to LS. Bundles in LS are more parallel, show less variation in orientation, and are more densely packed than in CS. There was a linear correlation between mean skin score and orientation ratio. Local skin score was not linearly correlated to orientation ratio. Our findings suggest that nonLS dermis without clinical sclerosis already shows fibrotic characteristics. Both techniques were easy to use and suitable for objectifying dermal fibrosis in scleroderma lesions. FFT is more accurate and reproducible than the laser scatter method and allows simultaneous pathological evaluation of the location of the analyzed tissue sections. Future studies will need to focus on the correlation between clinical disease severity and collagen bundle characteristics. (Lab Invest 2000, 80:1281-1289).
\end{abstract}

\begin{abstract}
S cleroderma forms a spectrum of connective tis$\int$ sue disorders ranging from systemic sclerosis at one end to the so-called overlap syndromes in the middle, and localized scleroderma, also known as morphea, at the other end (Haustein and Anderegg, 1998). The pathogenesis is as yet unknown, but the activation of $\mathrm{T}$ lymphocytes, possibly through abnormal T cell receptors and super- and/or auto antigens, seems to play a major role in the increase of collagen and the vascular damage so typical of sclerodermic lesions (Furst and Clements, 1996). Moreover, there is a genetic predisposition for scleroderma in individuals with HLA-DR5, -DR8, and -DR11 genotypes (Okun et al, 1988). Damage to genetic material might be another cause of scleroderma (Furst and Clements, 1996). Toxic agents related to systemic sclerosis are aromatic hydrocarbons, silica, and drugs like penicil-
\end{abstract}

Received March 28, 2000.

Address reprint requests to: Dr. Henry J.C. de Vries, Department of Dermatology, Academic Medical Center, P.O. Box 227001100 DE, Amsterdam, The Netherlands. Fax $3120 \quad 566$ 9152; E-mail: h.j.devries@amc.uva.nl lamine and L-tryptophan. Recently, mast cells (Akimoto et al, 1998) and fetal stem cells in the maternal circulation after childbirth have been addressed as possible determinants in the development of scleroderma (Artlett et al, 1998).

One of the main criteria of scleroderma is fibrosis of the dermal layer. Early clinical manifestations consist of ill-defined plaques of edema and erythema. Gradually, the distinct dermatological entity with sharply defined plaques of thickened, shiny, white, tanned, smooth centers arise. The margins can be characterized by a lilac ring that implies inflammation and probable progression of the disease.

Histologically, sclerosis is characterized in the early stages by an inflammatory cellular infiltrate composed of lymphocytes, macrophages, mast cells, eosinophils, and plasma cells (Guitart et al, 1996). With time, collagen bundles become broadened and closely packed and the dermis as a whole thickens. In normal skin, collagen bundles show a three-dimensional basket-weave pattern, whereas in scleroderma lesions, the collagen bundles are oriented parallel to the epidermis (Hamilton, 1967; Guitart et al,1996). The 
subcutaneous and periappendicular fat, as well as skin appendages, are progressively lost and replaced by collagen bundles.

To determine the severity of sclerodermic lesions, the skin score is a widely used subjective measure. It represents the induration or loss of elasticity assessed in a body area. The local skin score is estimated by pinching a skin fold in one area and scoring it on a scale of 0 to 3, indicating, respectively, no sclerosis to severe sclerosis (Enomoto et al, 1999). The mean skin score is the average of the total body surface subdivided into 74 areas. Because the clinical course of scleroderma is unpredictable and the treatment is still the subject of clinical trials, objective parameters are needed for reporting disease progression and to evaluate therapies. Several devices to objectify skin elasticity in sclerodermic lesions have been developed. One of the devices used in our clinic, called the cutometer, exerts a controlled vacuum over the skin. The extent of deformation is determined by a noncontact optical measuring system (Enomoto et al, 1996). Dermal thickness can be measured with high frequency ultrasound (Hoffmann et al, 1991) and nuclear magnetic resonance imaging (Richard et al, 1991). Objective histologic evaluation can be performed by measuring the amount of collagen in standard skin biopsy specimens. These methods consist of weighing and histometric methods (Dykes and Marks, 1977; Rodnan et al, 1979), which are inaccurate, and biochemical assays (Zachariae et al, 1989), which are elaborate.

In this paper we describe two techniques, the laser scatter method and the fast Fourier transform (FFT), for measuring the severity of dermal sclerosis based on the architecture of the collagen bundles. These two methods are compared with the mean and local skin score. The laser scatter method was developed by Yannas and Ferdman to register the grade of organization in scars of deep dermal wounds (Ferdman and Yannas, 1993). The overall orientation of collagen bundles in fibrotic tissue was quantified using the laser light scatter characteristics of fibrotic tissue. In a previous study, we used this method to evaluate the effect of different dermal substitutes in wound healing (de Vries et al, 1995a). Like mature scar tissue, sclerodermic lesions are also characterized by parallel collagen bundles (Okun et al, 1988; de Vries et al, 1995b).

The FFT is a mathematical analog of the laser scatter plot. It calculates orientation and spacing in an image. The periodicity and the orientation of structures, such as collagen bundles, can be represented as a power plot of the FFT of an image (Russ, 1995). Interpretation of the power plot is much easier and more accurate than trying to extract the same information from the original image, because all the spacings and orientations are effectively averaged in a frequency domain. The FFT allows measurement of spacing and orientation of collagen bundles, as well as a histopathologic evaluation of the same location. This simultaneous evaluation is not possible with the laser scatter method. FFT has been used for the evaluation of dermal architecture in scar tissue (van Zuijlen et al, 1998), and proves to be a simple, objective, and reproducible method for analyzing the severity of fibrosis in tissue sections from scleroderma lesions.

\section{Results}

\section{Patient Population}

Twenty patients, 17 women and 3 men, were included in the study (Table 1). A total of 35 biopsies were collected, both lesional $(n=23)$ and nonlesional $(n=12)$. The mean age of the scleroderma patient group was $46.6 \pm 16.2$ years. Disease duration at the time of the first biopsy collection was $24.4 \pm 23.6$ months. Eleven biopsies, collected from persons without fibrotic or sclerotic skin conditions, served as control.

\section{Histology}

Lesional sclerodermic skin (LS) (Fig. 1a) showed the typical densely packed arrangement of collagen bundles parallel to the epidermis. Skin appendages, like hair follicles and glandular structures, were absent. Dermal capillaries were characterized by thickened capillary walls. The dermal compartment as a whole was increased and subcutaneous fat decreased compared with control skin (CS).

In nonlesional sclerodermic skin (nonLS), the dermal thickness was increased to a lesser extent than in LS biopsies. Periappendicular fat was decreased, but skin appendages were present. It was difficult to evaluate the orientation of collagen bundles compared with LS and CS biopsies.

Unaffected CS (Fig. 1b) was characterized by dermal tissue with a randomly oriented, basket-weave network of collagen bundles. Skin appendages were abundantly present, surrounded with loosely arranged fat tissue.

\section{The Laser Scatter Method}

The dermis of LS (Fig. 2a) showed a higher orientation ratio than CS (Fig. 2b), respectively, $2.16 \pm 0.33$ and $1.70 \pm 0.35 ; p=0.005$ (Table 2). This indicates that the collagen bundles in sclerodermic skin show a more parallel alignment than do collagen bundles in normal dermis. No significant differences in orientation ratio were found between LS and nonLS (1.92 \pm 0.15$)$, nor between nonLS and CS.

\section{Fast Fourier Transform}

Because of the fluorescent properties of eosin, which stains the collagen bundles, the confocal laser scanning microscope selectively images these bundles without interference from the hematoxylin-stained structures (ie, nuclei). The highly parallel orientation of collagen bundles in LS biopsies can be appreciated 
Table 1. Patient and Biopsy Information

\begin{tabular}{|c|c|c|c|c|c|}
\hline Patient & $\operatorname{Sex}^{a}$ & $\begin{array}{c}\text { Age } \\
\text { (in years) }\end{array}$ & $\begin{array}{l}\text { Biopsy } \\
\text { type }^{b}\end{array}$ & $\begin{array}{l}\text { Disease duration } \\
\quad \text { (in months) }\end{array}$ & Perivascular inflammation \\
\hline \multirow[t]{2}{*}{1} & $f$ & 55 & Is & 58 & Absent \\
\hline & & & $\mathrm{nl}$ & 58 & Absent \\
\hline \multirow[t]{3}{*}{2} & $f$ & 29 & Is & 15 & Absent \\
\hline & & & Is & 48 & Trace \\
\hline & & & $\mathrm{nl}$ & 48 & Absent \\
\hline 3 & $f$ & 30 & Is & 9 & Present \\
\hline \multirow[t]{3}{*}{4} & $f$ & 69 & Is & 23 & Trace \\
\hline & & & Is & 47 & Trace \\
\hline & & & $\mathrm{nl}$ & 47 & Trace \\
\hline \multirow[t]{3}{*}{5} & $\mathrm{~m}$ & 44 & Is & 51 & Absent \\
\hline & & & Is & 68 & Trace \\
\hline & & & $\mathrm{nl}$ & 68 & Present \\
\hline 6 & $f$ & 51 & Is & 15 & Present \\
\hline \multirow[t]{3}{*}{7} & $f$ & 54 & Is & 48 & Absent \\
\hline & & & Is & 64 & Trace \\
\hline & & & $\mathrm{nl}$ & 64 & Trace \\
\hline 8 & $f$ & 12 & Is & 74 & $\begin{array}{l}\text { Present, with } \\
\text { eosinophiles }\end{array}$ \\
\hline 9 & $f$ & 12 & $\mathrm{nl}$ & 74 & Present \\
\hline 10 & $\mathrm{~m}$ & 41 & Is & 9 & Present \\
\hline 11 & $f$ & 32 & $\mathrm{nl}$ & 4 & Present \\
\hline 12 & $f$ & 32 & Is & 4 & Present \\
\hline \multirow[t]{2}{*}{13} & $f$ & 44 & Is & 1 & Trace \\
\hline & & & $\mathrm{nl}$ & 1 & Trace \\
\hline \multirow[t]{3}{*}{14} & $f$ & 40 & Is & 7 & Present \\
\hline & & & Is & 29 & Absent \\
\hline & & & $\mathrm{nl}$ & 29 & Trace \\
\hline 15 & $\mathrm{~m}$ & 85 & Is & 2 & Present \\
\hline \multirow[t]{2}{*}{16} & $f$ & 45 & Is & 19 & Trace \\
\hline & & & $\mathrm{nl}$ & 19 & Trace \\
\hline \multirow[t]{2}{*}{17} & $f$ & 67 & Is & 35 & Trace \\
\hline & & & $\mathrm{nl}$ & 35 & Trace \\
\hline 18 & $f$ & 67 & Is & 35 & Absent \\
\hline \multirow[t]{2}{*}{19} & $f$ & 28 & Is & 9 & $\begin{array}{l}\text { Present, with } \\
\text { eosinophiles }\end{array}$ \\
\hline & & & Is & 31 & Trace \\
\hline 20 & $f$ & 30 & $\mathrm{nl}$ & 31 & Present \\
\hline
\end{tabular}

${ }^{a} f=$ female, $m=$ male

${ }^{b}$ Is = lesional sclerodermic skin, $\mathrm{nl}=$ nonlesional sclerodermic skin.

(Fig. 3a), as well as the basket-weave structure of collagen bundles in the CS biopsies (Fig. 3b).

The power plot of an FFT of the LS biopsies showed two first-order maxima oriented along a line perpendicular to the collagen bundle orientation (Fig. 4a). The power plot of the CS biopsies showed an oval without obvious maxima, indicating a random orientation (Fig. 4b).

Results similar to those found with the laser scatter method were obtained with the FFT. LS showed a significantly higher orientation ratio than CS (respectively, $1.83 \pm 0.62$ and $1.38 \pm 0.15$; $p=0.002$; Table 2), and no significant difference was found in orientation ratio between LS and nonLS $(1.48 \pm 0.44)$, nor between nonLS and CS. With both techniques, the orientation ratio of the nonLS group was consistently lower than the LS and higher than the CS group.

\section{Orientation Variation and Collagen Bundle Spacing}

In both LS and nonLS biopsies, the orientation variation of the collagen bundles in relation to the epidermis was similar (respectively, $57.3 \pm 19.4$ and $57.2 \pm 29.0$; Table 3). In CS (73.8 \pm 15.0 ), the orientation variation of collagen bundles was significantly larger than LS $(p=0.03)$. No significant difference was found between CS and nonLS ( $p=$ $0.15)$. These findings indicate that in LS and nonLS, the orientation of collagen bundles can be found within a smaller range than in unaffected skin.

Bundle spacing represents the center-to-center distance between collagen bundles, and reflects the den- 


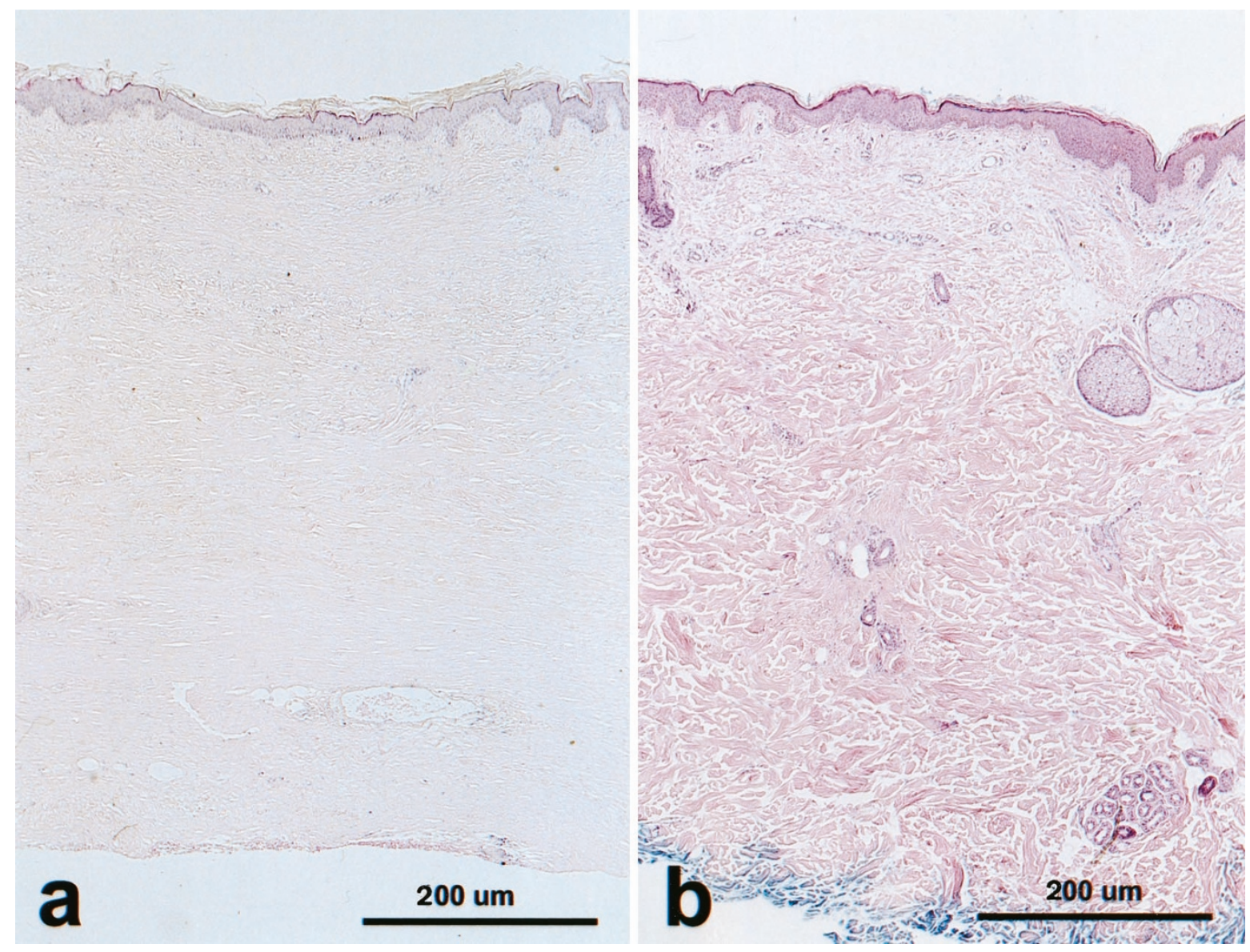

\section{Figure 1.}

Histology. a) Lesional sclerodermic skin (LS) with parallel, densely packed collagen bundles. Skin appendages are absent. b) Unaffected control skin (CS) shows randomly oriented, more loosely arranged collagen bundles. Skin appendages are present (hematoxylin-eosin staining).
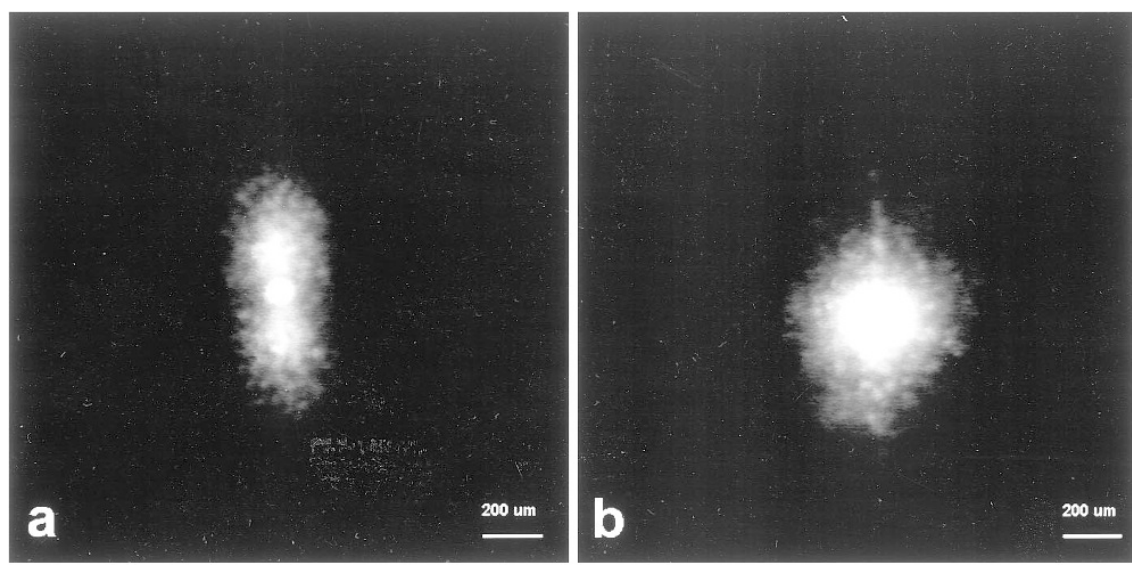

\section{Figure 2.}

Laser scatter images. a) LS produces an elongated scatter plot, indicating a higher orientation of collagen bundles than CS. b) CS produces a circular scatter plot caused by the randomly oriented collagen bundles.

sity in the dermal layer. A low spacing value correlates with high bundle density and vice versa. Bundle spacing was comparable for the LS and nonLS group (respectively, $15.7 \pm 5.6$ and $15.6 \pm 6.1$ ) but was higher in the control group (CS is $18.9 \pm 1.9$ ). There was a significant difference between LS and CS ( $p=0.03)$, but not between nonLS and CS ( $p=0.14)$. These data prove that collagen bundles in LS are more densely packed than in unaffected skin, and the bundle density in nonLS is increased. 
Table 2. Orientation Ratio Calculated with the Laser Scatter Method and Fast Fourier Transform (FFT)

\begin{tabular}{ccccc}
\hline Technique & & Lesional & Nonlesional & Control (CS) \\
\hline laser scatter & $\mathrm{n}$ & 11 & 5 & 11 \\
& $\mathrm{x} \pm \mathrm{SD}$ & $2.16 \pm 0.33 \#$ & $1.92 \pm 0.15$ & $1.70 \pm 0.35 \#$ \\
FFT & $\mathrm{n}$ & 24 & 11 & 11 \\
& $\mathrm{x} \pm \mathrm{SD}$ & $1.83 \pm 0.62^{*}$ & $1.48 \pm 0.44$ & $1.38 \pm 0.15^{\star}$ \\
\hline
\end{tabular}

$\# p=0.005,{ }^{*} p=0.002$
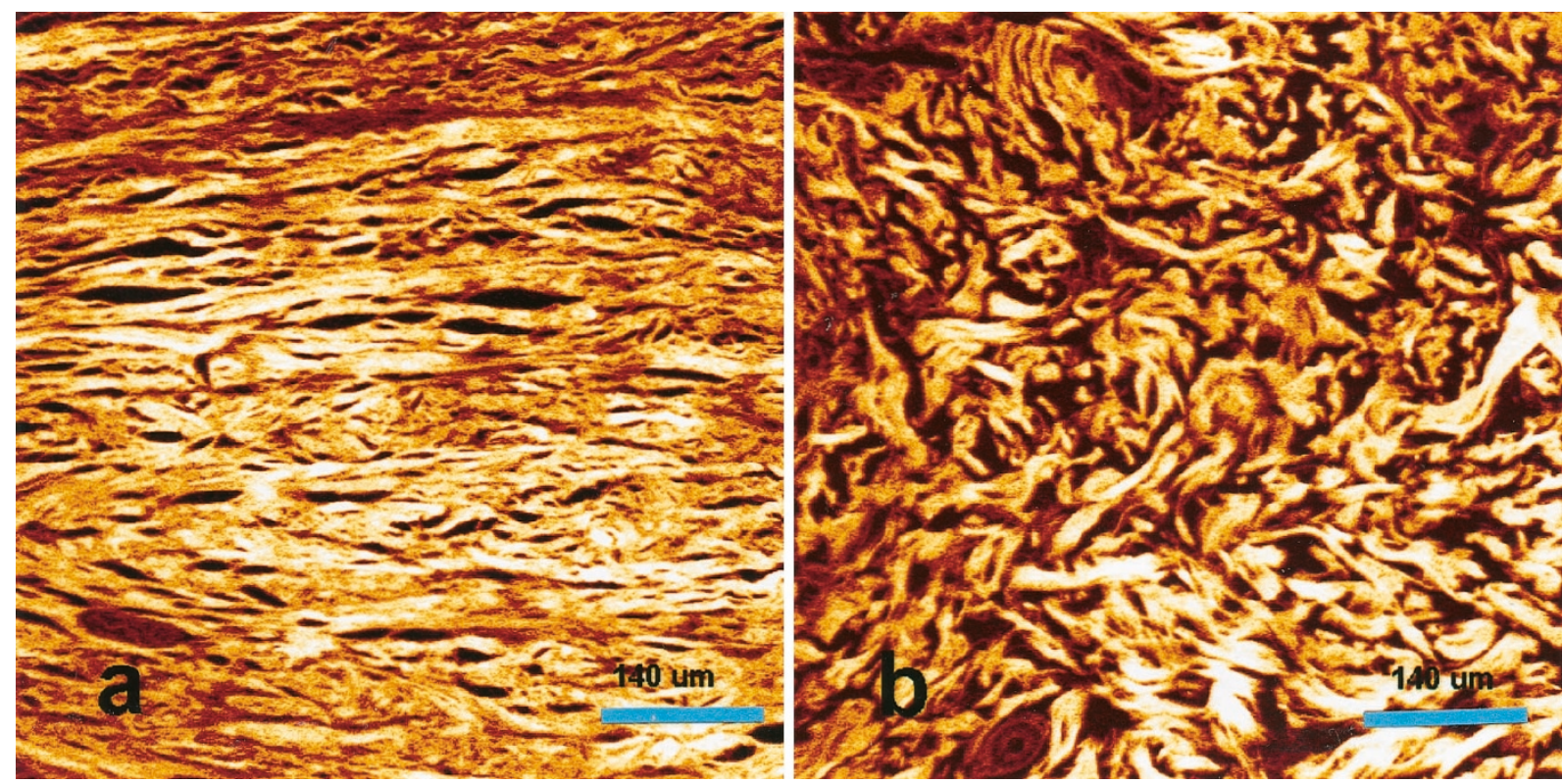

\section{Figure 3.}

Confocal laser scanning microscopy images. Because the fluorescent signal is from the eosin-stained collagen bundles exclusively, no hematoxylin stained (ie, cellular structures) are depicted. a) LS again shows parallel collagen bundles, in contrast to b), CS showing randomly oriented collagen bundles.

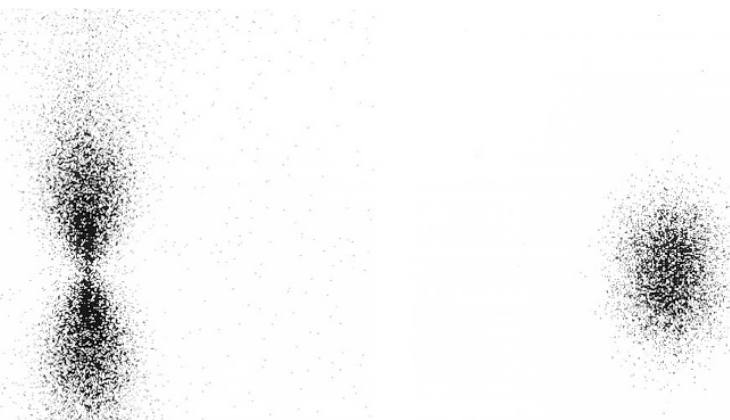

Figure 4.

First-order power plots from the fast Fourier transform (FFT). a) LS creates a power plot with a preferential distribution with two maxima. b) CS is characterized by a power plot with a circular and no preferential distribution pattern.

\section{Skin Score}

FFT data (orientation ratio, orientation variation, and bundle spacing) were related to the mean skin score (Table 4) and to the local skin score results from the corresponding biopsy areas (Table 5). Mean skin scores were divided into three groups: skin score 0 , skin scores 0 to 0.5 , and skin scores higher than
0.50. There was a linear correlation between orientation ratio and mean skin score group (respectively, $1.41 \pm 0.11,1.82 \pm 0.68$, and $1.95 \pm 0.74$; Table 4). With Spearman's rank test, a 0.5 correlation coefficient was found and is significant at the 0.01 level (two-tailed). No significant relationships were found for orientation variation and bundle spacing. 
Table 3. Orientation Variation of Collagen Bundles Relative to the Epidermis and Spacing of Collagen Bundles as Calculated with the FFT

\begin{tabular}{lccc}
\hline \multicolumn{1}{c}{ Parameter } & Lesional & Nonlesional & Control \\
\hline $\mathrm{n}$ & 24 & 10 & 11 \\
Orientation variation $\left(\mathrm{x} \pm \mathrm{SD}\right.$ in $\left.^{\circ}\right)$ & $57.3 \pm 19.4 \#$ & $57.2 \pm 29.0 \dagger$ & $73.8 \pm 15.0 \# \dagger$ \\
Bundle spacing $(\mathrm{x} \pm \mathrm{SD}$ in $\mu \mathrm{m})$ & $15.7 \pm 5.6^{*}$ & $15.6 \pm 6.1 \S$ & $18.9 \pm 1.9^{*} \S$ \\
\hline
\end{tabular}

$\# p=0.03,{ }^{*} p=0.03, \dagger p=0.15, \S p=0.14$.

Table 4. Mean Skin Score Relative to Orientation Ratio, Orientation Variation, and Bundle Spacing as Measured with the FFT

\begin{tabular}{cccl}
\hline Mean skin score & \multicolumn{1}{c}{$\begin{array}{l}0>0.5 \\
(\mathrm{n}=16)\end{array}$} & \multicolumn{1}{c}{$<0.5(\mathrm{n}=7)$} \\
\hline Orientation ratio $(\mathrm{x} \pm \mathrm{sD})$ & $1.41 \pm 0.11 \#$ & $1.82 \pm 0.68 \#^{\star}$ & $1.95 \pm 0.74^{\star}$ \\
Orientation variation $(\mathrm{x} \pm \mathrm{sD}$ in & $\circ$ \\
Bundle spacing $(\mathrm{x} \pm \mathrm{SD}$ in $\mu \mathrm{m})$ & $63.0 \pm 25.8$ & $54.8 \pm 23.5$ & $57.8 \pm 19.0$ \\
\hline
\end{tabular}

$\# p=0.01,{ }^{*} p=0.2$.

Table 5. Local Skin Score Relative to Orientation Ratio, Orientation Variation, and Bundle Spacing as Measured with the FFT

\begin{tabular}{|c|c|c|c|c|}
\hline Local skin score & $0(n=10)$ & $1(n=7)$ & $2(n=6)$ & $3(n=5)$ \\
\hline Orientatio & $1.36 \pm 0.14 \#^{\star} \S$ & $2.08 \pm 0.83 \#$ & $1.70 \pm 0.24^{*}$ & $1.95 \pm 0.88 \S$ \\
\hline Orientation variation $\left(\mathrm{X} \pm \mathrm{SD}\right.$ in $\left.{ }^{\circ}\right)$ & $50.9 \pm 31.4$ & $66.3 \pm 13.6$ & $46.3 \pm 22.8$ & $52.4 \pm 15.2$ \\
\hline Bundle spacing $(\mathrm{x} \pm \mathrm{SD}$ in $\mu \mathrm{m})$ & $16.8 \pm 13.0$ & $16.5 \pm 2.2$ & $14.0 \pm 7.6$ & $16.1 \pm 6.4$ \\
\hline
\end{tabular}

$\# p=0.02,{ }^{*} p=0.004, \S p=0.07$

The orientation ratio in the local skin score 0 group $(1.36 \pm 0.14)$ differed significantly from the local skin score 1 to 3 groups (respectively, $2.08 \pm 0.83$, $p=0.02 ; 1.70 \pm 0.24, p=0.004 ;$ and $1.95 \pm 0.88$, $p=0.07$; Table 5). No linear correlation was observed between orientation ratio and the severity of skin scores, but the Spearman's rank correlation coefficient was 0.6 with a 0.01 significancy level (two-tailed). No significant relationships were found for orientation variation and bundle spacing.

\section{Discussion}

In skin biology, processes like aging and scar formation alter the dermal organization. Skin diseases like scleroderma and lichen sclerosus affect the dermal collagen bundle architecture. The two analysis methods described in this article, the laser scatter method and the FFT, may provide information on the development, gradation, and prognosis of sclerotic processes. The laser scatter method was previously used to evaluate scar formation in experimental wounds in guinea pigs (Ferdman and Yannas, 1993) and in humans (de Vries et al, 1995a). We demonstrate the use of the FFT to quantify the architecture of dermis at the level of collagen bundles.

The laser scatter method and the FFT proved to be easy-to-use tools to discriminate between normally organized and sclerotic dermal tissue. Tissue slides from routine formaldehyde-fixed skin biopsies suffice. For the FFT, a personal computer with commercially available image analysis software and a fluorescence microscope equipped with a digital camera with frame grabber are the main research tools. Normal bright field microscopic images of hematoxylin-eosinstained material are not really suitable for FFT analysis because the hematoxylin-stained nuclei dominate the image and are consequently conspicuous in the power plot. Using the fluorescent properties of eosin eliminates this problem. We used a confocal microscope for data acquisition because it was available and convenient for digitizing the images, but a normal fluorescence microscope works just as well. The laser scatter method can be performed with a standard laser source, a simple optical bench arrangement plus a digital camera, a personal computer, and image analysis software.

The FFT proved to be a more accurate method for the evaluation of fibrosis than the laser scatter method. The latter does not allow exact positioning of the laser beam, and simultaneous microscopic monitoring of the target area is not possible. The FFT is performed on digitized microscopic images that allow the investigator to accurately determine the position and size of the area to be analyzed.

In both the laser scatter method and the FFT, LS showed a more extensive parallel orientation of collagen bundles than unaffected CS. This was revealed by the orientation ratio observations (Table 2). The orientation variation, that is, the variation of the bundle orientation in relation to the epidermis, was signifi- 
cantly reduced in the LS group compared with the CS group (Table 3 ). This finding indicates that there is less variation in the organization of collagen bundles in LS. Bundle spacing, or center-to-center distance of the collagen bundles, was significantly lower in LS dermis compared with CS dermis (Table 3). Thus it can be concluded that the collagen bundles in sclerotic skin are more densely packed than in unaffected CS.

Using FFT and the laser scatter method, we found indications that in the dermal layer of clinically unsuspected nonLS, the collagen bundle structure shows some alterations that indicate early fibrosis. Although the results are not significant because of the small number of samples, the orientation ratio found with both methods showed increased alignment of collagen bundles in nonLS biopsies compared with CS biopsies, but less alignment than in LS biopsies. More evidence of early changes in nonLS was supplied by the observations on orientation variation and bundle spacing. For nonLS as well as for LS, both parameters were of similar magnitude. The orientation variation and bundle spacing values of nonLS also differed from the CS group, although not significantly. It is assumed that fibrosis in scleroderma is a gradual process. Our findings support this assumption; nonLS without the clinical signs of sclerosis already shows some dermal characteristics similar to sclerodermic skin.

Orientation ratio, orientation variation, and bundle spacing as measured with the FFT were related to the skin score data. A positive linear correlation was found between mean skin score and the orientation ratio (Table 4). Although the correlation coefficient was not strong (Spearman's rank 0.5, $p<0.01$, two-tailed), this finding supports our hypothesis that the objective parameters as described in this article can be related to the overall clinical severity of sclerodermic skin. No correlation was found among orientation variation, bundle spacing, and local or mean skin score. No linear relationship between local skin score and FFT orientation ratio was found, but Spearman's rank test detected a significant correlation coefficient of 0.6 ( $p<0.01$, two-tailed) (Table 5).

That only weak correlations were detected between skin score values and orientation ratio could be attributed to the small sample size of the skin score groups, which would also explain the large standard deviation. It also seems that the clinical skin scores are not equidistant. In a previous study, skin elasticity values were compared to skin scores (Enomoto et al, 1996). The difference in skin elasticity between skin score 0 (normal skin) and skin score 1 was larger than the difference between skin score 1 and 2, or between skin score 2 and 3 . The same phenomenon seems to take place in the observations on collagen bundle characteristics. In the skin score 1 group, collagen bundles show distinctive parallel orientation; in skin score groups 2 and 3 , the parallel orientation is only slightly increased. The subjective discrimination made between skin scores 1 to 3 could be caused by aspects other than collagen bundle characteristics, for example, dermal edema. Using high-frequency echography as a clinical parameter instead of the subjective skin score method might reveal more appropriate correlations between the collagen bundle characteristics and disease severity.

For orientation variation or bundle spacing, no significant differences were found between the various skin score groups. These findings could be attributed to the substantial within- or between-observer variation that characterizes and hampers the skin score method (Pope et al, 1995). For day-to-day clinical use, it will remain a first choice technique for assessing sclerosis, but when greater accuracy is required, the skin score method does not suffice.

In conclusion, both methods are suitable for objectifying collagen bundle structure and discriminating between fibrotic dermis and normal organized dermis, but the FFT proves to be more accurate and reproducible than the laser scatter method. Future studies will need to focus on the relationship between clinical disease severity and collagen bundle characteristics, possibly with more accurate methods to score disease severity, like high frequency echography.

\section{Materials and Methods}

Punch biopsies $(4 \mathrm{~mm})$ were collected from patients with a histologically and clinically confirmed systemic scleroderma diagnosis. Biopsies from patients without a fibrotic or sclerotic skin condition served as controls. Three groups were formed: 1) lesional scleroderma skin, and 2) nonlesional scleroderma skin, both derived from scleroderma patients; and 3) control skin from patients without a fibrotic or sclerotic skin condition. Tissue sections $(7 \mu \mathrm{m})$ were cut from formalinfixed, paraffin-embedded biopsies and stained with hematoxylin-eosin.

\section{The Laser Scatter Method}

Because of its wave characteristics, monochromatic light passing through a fine grid of parallel lines will be diffracted in preferential directions. When projected on a screen, a specific pattern of light peaks will occur perpendicular to the direction of the grid lines. A tissue section from dermal tissue can be interpreted as a grid. Normal dermis with its randomly oriented collagen bundles diffracts light in no preferential direction, causing a circular scatter pattern. The parallel collagen bundles in sclerotic tissue cause a light scatter pattern with a preferential direction perpendicular to the direction of these fibers. As a result, an elongated ovalshaped scatter pattern will occur.

The laser scatter method was performed as previously described (de Vries et al, 1995a). In short, a 3-mW helium/neon laser (Uniphase, Manteca, California) was mounted on an optical bench. To create a gaussian beam of suitable diameter $( \pm 100 \mu \mathrm{m})$ a spatial filter was constructed from two achromatic convex lenses with different focal lengths, and a 100- $\mu \mathrm{m}$ pinhole. A slide holder and a white paper screen were placed in line with the laser, and the scatter patterns were recorded with a black and white CCD camera (model VC-2512; Sanyo, Japan) con- 
nected to a computer image analyzer (Quantimet 500; Leica, Cambridge, United Kingdom). The light threshold of the image analyzer was standardized, and the maximum length and maximum breadth of each scatter pattern were automatically calculated.

\section{The Fast Fourier Transform}

Images $(794 \times 794 \mu \mathrm{m})$ were acquired with a confocal laser scanning microscope (Leica Micro Optics, Heidelberg, Germany) with a $6.3 \times 0.2$ objective and a 50 $\mu \mathrm{m}$ pinhole using the fluorescent properties of eosin (excitation wave length $488 \mathrm{~nm}$, emission wave length $>580 \mu \mathrm{m})$. This ensured a constant contrastbrightness index for all images independent of staining, and a constant optical section thickness (approximately $2 \mu \mathrm{m})$. This also guaranteed that the hematoxylin-stained nuclei, which dominate a conventional bright field image, were not imaged at all. The epidermis was always oriented parallel to the $x$-axis of the image.

Analysis of the images was performed with the FFT module of the Leica Qwin image analysis software (Leica). From each image, the FFT and its first-order power plots were calculated to provide an estimate of the orientation ratio in the image, the orientation variation, and the bundle spacing (Fig. 5). The orientation ratio corresponds to the length to breadth ratio of the first-order maximum of the power plot. Randomly oriented collagen bundles will evoke an orientation ratio approaching 1 , parallel collagen bundles a ratio greater than 1 . The orientation variation corre-

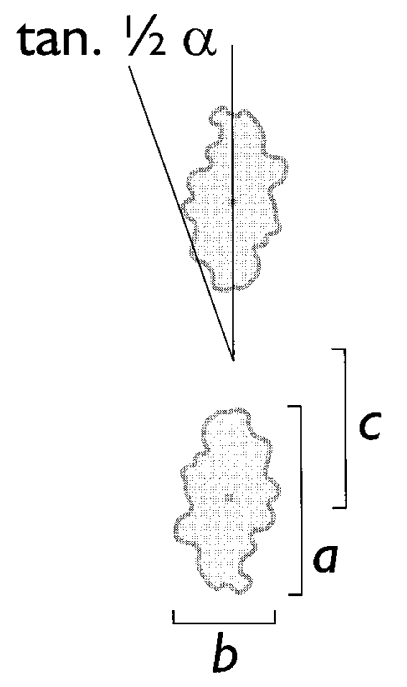

\section{Figure 5.}

Interpretation of the Fourier power plots. Confocal scanning laser microscopy images of the dermis were used to calculate the FFT and corresponding first-order power plots. The orientation ratio corresponds to the ratio $a / b$. Orientation variation corresponds to the angle tan $1 / 2 \alpha$ formed by the two lines, as shown. Bundle spacing corresponds to the distance $c$ between the center of the power plot and the centroid of the first-order maximum. $a$, Length of the first-order maximum; $b$, breadth of the first-order maximum; $c$, distance between the center of the power plot and the centroid of the first-order maximum. The two converging lines are a line through the center of the power plot and the centroid of the first-order maximum, and a line through the center of the power plot and along the edge of the first-order maximum. sponds to the angle over which the collagen bundles are oriented in the skin; in the power plot of an image, it is the angle between the tangent along the first-order maximum and the center of the power plot (Fig. 5). Bundle spacing corresponds to the reciprocal of the distance from the center of the power plot to the center of the first-order maximum.

\section{Skin Score}

Results from the laser scatter method and FFT were compared with the local skin score obtained in the skin region where the punch biopsy was taken. Skin score was assessed by a trained observer by palpation using the Rodnan modification for assessing cutaneous sclerosis on a 0 to 3 scale $(0=$ no sclerosis, $1=$ mild, $2=$ moderate, and $3=$ severe), as previously described (Enomoto et al, 1996). The mean skin score is the sum of the 74 local skin scores divided by the number of regions $(n=74)$. For comparison with collagen bundles, the continuum of mean skin scores was divided into three groups:1) mean skin score 0 ( $n=16)$; 2) mean skin score 0.01 to $0.49(n=12)$; and 3) mean skin score 0.50 to $2.00(n=7)$.

All statistical differences were tested with the unpaired Student's $t$ test. The two-tailed Spearman's rank test was used to detect a correlation between two groups.

\section{References}

Akimoto S, Ishikawa O, Igarashi Y, Kurosawa M, and Miyachi Y (1998). Dermal mast cells in scleroderma: Their skin density, tryptase/chymase phenotypes, and degranulation. $\mathrm{Br} \mathrm{J}$ Dermatol 138:399-406.

Artlett CM, Smith JB, and Jimenez SA (1998). Identification of fetal DNA and cells in skin lesions from women with systemic sclerosis. N Engl J Med 338:1186-1191.

de Vries HJ, Middelkoop E, van Heemstra-Hoen M, Wildevuur $\mathrm{CH}$, and Westerhof W. (1995a). Stromal cells from subcutaneous adipose tissue seeded in a native collagen/ elastin dermal substitute reduce wound contraction in full thickness skin defects. Lab Invest 73:532-540.

de Vries HJ, Zeegelaar JE, Middelkoop E, Gijsbers G, Van Marle J, Wildevuur CH, and Westerhof W. (1995b). Reduced wound contraction and scar formation in punch biopsy wounds. Native collagen dermal substitutes. A clinical study. Br J Dermatol 132:690-697.

Dykes PJ and Marks R (1977). Measurement of skin thickness: A comparison of two in vivo techniques with a conventional histometric method. J Invest Dermatol 69:275278.

Enomoto DN, Mekkes JR, Bossuyt PM, Hoekzema R, and Bos JD (1996). Quantification of cutaneous sclerosis with a skin elasticity meter in patients with generalized scleroderma. J Am Acad Dermatol 35:381-387.

Enomoto DN, Mekkes JR, Bossuyt PM, Yong SL, Out TA, Hoekzema R, de Rie MA, Schellekens PT, ten Berge IJ, de Borgie CA, and Bos JD (1999). Treatment of patients with systemic sclerosis with extracorporeal photochemotherapy (photopheresis). J Am Acad Dermatol 41:915-922. 
Ferdman AG and Yannas IV (1993). Scattering of light from histologic sections: A new method for the analysis of connective tissue. J Invest Dermatol 100:710-716.

Furst DE and Clements PJ (1996). Pathogenesis, Fusion (Summary). In: Clements PJ and Furst DE, editors. Systemic sclerosis. Baltimore: Williams and Wilkins, 275-284.

Guitart J, Micali G, and Solomon LM (1996). Localized scleroderma. In: Clements PJ and Furst DE, editors. Systemic sclerosis. Baltimore: Williams and Wilkins, 65-79.

Hamilton M (1967). Lupus erythematosus and scleroderma. In: Hamilton M, editor. Dermatopathology. New York: Harper and Row, 719-762.

Haustein UF and Anderegg U (1998). Pathophysiology of scleroderma: An update. J Eur Acad Derm Venereol 11:1-8.

Hoffmann K, Gerbaulet U, el-Gammal S, and Altmeyer P (1991). 20-MHz B-mode ultrasound in monitoring the course of localized scleroderma (morphea). Acta Derm Venereol 164:3-16.

Okun MR, Edelstein LM, and Fisher BK (1988). Scars. In: Okun MR, Edelstein LM, and Fisher BK, editors. Gross and microscopic pathology of the skin. Canton: Dermatopathology Foundation Press, 210-211.

Pope JE, Baron M, Bellamy N, Campbell J, Carette S, Chalmers I, Dales P, Hanly J, Kaminska EA, and Lee P (1995). Variability of skin scores and clinical measurements in scleroderma. J Rheumatol 22:1271-1276.
Richard S, Querleux B, Bittoun J, Idy-Peretti I, Jolivet O, Cermakova E, and Leveque JL (1991). In vivo proton relaxation times analysis of the skin layers by magnetic resonance imaging. J Invest Dermatol 97:120-125.

Rodnan GP, Lipinski E, and Luksick J (1979). Skin thickness and collagen content in progressive systemic sclerosis and localized scleroderma. Arthritis Rheum 22:130140.

Russ JC (1995). Processing images in frequency space. In: Russ JC, editor. The image processing handbook. Boca Raton: CRC Press, 283-346.

van Zuijlen PPM, van Leeuwen RTJ, Lamme EN, de Vries HJ, Taal LA, and Middelkoop E (1998). Assessment of collagen bundle orientation in scar tissue by means of the fast Fourier transform and laser scattering (abstract). Wound Repair Regen 6:A493.

Zachariae H, Halkier-Sorensen L, and Heickendorff L (1989). Serum aminoterminal propeptide of type III procollagen in progressive systemic sclerosis and localized scleroderma. Acta Derm Venereol 69:66-70. 